\title{
Effect of Ascorbic Acid and Etiolation on Antioxidant Enzyme Activity and Phenylpropanoid Metabolism during Shoot Regeneration from Cut Ends of
}

\section{Tomato Stems}

\author{
Masahumi Johkan**, Yoshihiro Imahori, Hajime Furukawa, Kazuhiko Mitsukuri, \\ Satoshi Yamasaki, Hideyuki Tanaka and Masayuki Oda*
}

Graduate school of life and environmental Sciences, Osaka Prefecture University, Sakai 599-8531, Japan

\begin{abstract}
We investigated the effects of ascorbic acid (AsA) or etiolation treatments on the regeneration of shoots from cut ends of tomato stems. For the AsA treatment, AsA solution was applied to leaves of tomato plants immediately after cutting the main stem. The etiolation treatment consisted of covering the cut stem with an aluminum cap. When applied individually, the AsA or etiolation treatments resulted in increased numbers of shoots regenerated from the cut stem. However, application of both treatments simultaneously did not have a synergetic effect on the number of shoots regenerated. To clarify the mechanisms by which these treatments promoted shoot regeneration, we determined the effects of AsA or etiolation treatments on scavenging of reactive oxygen species (ROS) by antioxidant enzymes and phenylpropanoid metabolism in the cut ends of tomato stems during the shoot regeneration process. In this study, activities of antioxidant enzymes (superoxide dismutase, ascorbate peroxidase, catalase) were higher in most of the stems that received AsA or etiolation treatments, compared with those of the control. In addition, compared with the control, plants treated with individual AsA or etiolation had lower levels of hydrogen peroxide, malondialdehyde, and phenols, and lower phenylalanine ammonia-lyase activity. These results indicated that the promotive effects of individual AsA or etiolation treatments on shoot regeneration from cut tomato stems can be attributed to the same mechanism, which involves the high activity of antioxidant enzyme, suppression of oxidative stress and phenol accumulation.
\end{abstract}

Key Words: in vivo, oxidative stress, phenols, vegetative propagation.

\section{Introduction}

In tomatoes, the most common method of mass propagation is reproduction by seed. This method has several advantages over vegetative reproduction methods, in that reproduction by seeds yields many virusfree progeny with the same genotype, and the storability of seeds is excellent. However, vegetative propagation does not require a process of genetic fixation, so it can be used for rapid release of cultivars with resistance to new pathogenic fungi or those that are currently in vogue with consumers. Indeed, the tomato cultivar 'Koshinoruby', which has high sugar content, has been reproduced via vegetative reproduction since its registration in 1992.

Received; May 10, 2010. Accepted; August 1, 2010.

* Corresponding author (E-mail: moda@plant.osakafu-u.ac.jp).

** Present address: Graduate School of Horticulture, Chiba University, Matsudo 271-8510, Japan.
However, because of the low efficiency of vegetative propagation, the distribution of cultivars reproduced by such methods is restricted. Tissue culture in vitro is an effective method for mass propagation of clones, but it is expensive and often generates somatic mutations (Padmanabhan et al., 1974).

Inexpensive methods of vegetative mass propagation in vivo have been reported for tomato (Oda et al., 2008) and sweet pepper (Shirai and Hagimori, 2004a, b, c). In tomato, about 65 lateral shoots were generated from the main and the second stem, but the multiplication rate was restricted by the number of axils (Oda et al., 2008). To increase the multiplication rate, regeneration of adventitious buds from the cut ends of tomato stems was established as a new approach in vegetative propagation (Harada et al., 2005). Recently, several methods that promote shoot regeneration from the cut ends of tomato stems have been reported. For example, foliar application of ascorbic acid (AsA) resulted in a 1.4-times increase 
in shoot regeneration compared with the control (Johkan et al., 2008b), and an etiolation treatment of the cut ends increased the regeneration rate to at least double that of the control (Johkan et al., 2008a, c, 2009). However, the synergetic effect of simultaneous AsA and etiolation treatments on promoting shoot regeneration was not investigated.

The mechanism responsible for the increase in the shoot regeneration from the cut ends of tomato stems treated with AsA or etiolation has also remained unknown. From the results of our previous studies, we thought that AsA treatment affected the scavenging of reactive oxygen species (ROS) (Johkan et al., 2008b) and that etiolation treatment suppressed synthesis of phenols (Johkan et al., 2008a, c). Scavenging of ROS by antioxidant enzymes (Libik et al., 2005; Qin et al., 2005; Tian et al., 2003) and suppression of phenolic oxidation, which suppressed plant regeneration from explants (Gupta and Datta, 2003; Mitsukuri et al., 2009; Sharma and Singh, 2002), were reported as key factors for efficient in vitro micropropagation. However, in the context of plant regeneration, antioxidant enzymes activities and phenylpropanoid metabolism related to the accumulation of phenols have rarely been studied at the same time.

In this study, we determined the effects of AsA treatment, etiolation treatment, and simultaneous treatment with AsA and etiolation on shoot regeneration from the cut ends of tomato stems. We also explored the activities of antioxidant enzymes and phenylpropanoid metabolism in the cut ends of tomato stems. Finally, we discuss the functions of these promotive treatments in shoot regeneration from cut tomato stems.

\section{Materials and Methods}

\section{Plant materials}

Germinated seeds of tomato plants (Solanum lycopersicum L., cv. Momotaro, Takii seed Co. Ltd., Kyoto, Japan) were sown in a 72 -cell-tray $(50 \mathrm{~mL} /$ cell $)$ filled with a $2: 1(\mathrm{v} / \mathrm{v})$ mixture of peat moss (Super cell top, Sakata seed Co. Ltd., Yokohama, Japan) and vermiculite (Nittai Co. Ltd., Osaka, Japan). The seedlings were grown in a greenhouse with a light transmissivity of $55 \%$, and supplied with nutrient solution containing $4.6 \mathrm{~N}, 1.3 \mathrm{P}, 2.2 \mathrm{~K}, 1.1 \mathrm{Ca}$, and 0.4 $\mathrm{Mg}$ (in $\mathrm{mmol} \cdot \mathrm{L}^{-1}$ ). When the 6 th true leaf had unfolded, the main stems were cut between the 2nd and 3rd true leaves with a sharp razor blade. For the ascorbic acid (AsA) treatment, $25 \mathrm{~mL}$ of a $100 \mathrm{mg} \cdot \mathrm{L}^{-1}$ sodium ascorbate (Wako Chemical Co. Ltd., Osaka, Japan) solution was sprayed on to the tomato plant immediately after cutting of the main stem. For the etiolation treatment, the cut stem was covered with an aluminum cap $(\Phi 30 \times 40 \mathrm{~mm})$ immediately after cutting, and the cap was removed when adventitious buds were observed on the cut surfaces of main stems. The AsA application was repeated weekly throughout the experiment. All lateral shoots were excised at their bases before they reached $5 \mathrm{~mm}$ in length.

\section{Synergetic effect of AsA and etiolation on shoot regeneration (Exp. 1)}

Tomato seeds were sown on 15 February 2006. Stems were cut and AsA-only, etiolation-only and simultaneous AsA+etiolation treatments were carried out on 27 April. Shoots regenerated from callus on the cut surface of stems were harvested 9 weeks after cutting (WAC) when the regenerated shoots reached $5 \mathrm{~cm}$ in length. Each treatment consisted of 12 plants. The average maximum and minimum air temperatures after cutting of stems were 29.7 and $18.1^{\circ} \mathrm{C}$, respectively.

Antioxidant enzymes activities and oxidative stress affected by AsA or etiolation (Exp. 2)

Tomato seedlings were sown on 3 January 2007. Stems were cut and AsA or etiolation treatments were carried out on 21 February 2007. The cut stems were sampled by cutting $1 \mathrm{~cm}$ below the top of the cut surfaces at 0 , $1,2,3$, and 4 WAC. These stems were stored at $-80^{\circ} \mathrm{C}$ until biochemical and enzymatic analyses were carried out, as described for Exp. 2 and Exp. 3. The average maximum and minimum air temperatures after cutting of stems were 28.3 and $15.1^{\circ} \mathrm{C}$, respectively.

Extractions of superoxide dismutase (SOD; EC 1.15.1.1.), ascorbate peroxidase (APX; EC 1.11.1.11.), and catalase (CAT; EC 1.11.1.6.) and determinations of their activities were carried out according to the methods of Kawakami et al. (2000) and Nakano and Asada (1981), respectively. Protein content was determined by the method of Bradford (1976), with bovine serum albumin (Wako Chemical Co. Ltd.) as the standard. $\mathrm{H}_{2} \mathrm{O}_{2}$ was extracted and quantified by the method of Okuda et al. (1991). Malondialdehyde (MDA) was extracted and quantified by the method of Dipierro and De Leonardis (1997).

\section{Phenylpropanoid metabolism affected by AsA or etiolation (Exp. 3)}

Plant samples were prepared as described for Exp. 2. Extractions of phenylalanine ammonia-lyase (PAL; EC 4.3.1.5.), polyphenol oxidase (PPO; EC 1.10.3.1.), and peroxidase (POX; EC 1.11.1.7.), and determinations of their activities were carried out according to the methods of Feng et al. (2006), Murata et al. (1995), and Dogan et al. (2007), respectively. Protein content was quantified using the method of Bradford (1976), with bovine serum albumin as the standard. Phenols were extracted with $80 \%$ methanol and quantified as described by Singleton and Rossi (1965).

\section{Statistical analysis}

Tukey's multiple range tests were used to test differences among more than two means using MEPHAS statistical software (Osaka University, Suita, Japan. 
http://www.geninfo.osaka-u.ac.jp/MEPHAS). Differences were considered significant at $P<0.05$.

\section{Results}

Synergetic effect of AsA and etiolation on shoot regeneration (Exp. 1)

When applied individually, AsA or etiolation treatments promoted formation of callus and adventitious buds, and increased the shoot number (Table 1). At 9 WAC, $83 \%$ of control plants showed callus formation and $17 \%$ formed adventitious buds. In the AsA-only treatment, $100 \%$ of plants formed calluses and $83 \%$ formed adventitious buds. All of the etiolated plants formed calluses and adventitious buds, regardless of AsA treatment. The mean value of shoots per plant was 0.1 in the control, 13.1 in AsA-treated plants, and 33.3 in etiolated plants. However, simultaneous treatment with AsA and etiolation did not have a synergetic effect on shoot regeneration; plants that received simultaneous AsA and etiolation treatments had no more shoots than those which received the etiolation treatment alone.

\section{Antioxidant enzymes activities and oxidative stress} affected by AsA or etiolation (Exp. 2)

Plants treated with AsA or etiolation showed higher activities of antioxidant enzymes in their cut stems than those of the control (Fig. 1). SOD activity increased immediately in all plants at $1 \mathrm{WAC}$, and remained at a higher level than that at 0 WAC (Fig. 1A). APX activity increased significantly at $1 \mathrm{WAC}$ in etiolated tomato stems and at 2 WAC in AsA-treated tomato stems. However, APX activity in control stems decreased at 1 WAC and remained at low levels throughout the experimental period (Fig. 1B). CAT activity in tomato stems treated with AsA or etiolation significantly increased at 1 WAC and remained at high levels throughout the experimental period, whereas that in control stems remained at pre-cutting levels (Fig. 1C).

We also determined $\mathrm{H}_{2} \mathrm{O}_{2}$ and MDA contents in cut ends of tomato stems to gauge the level of oxidative stress (Fig. 2). Cut ends of AsA- or etiolation-treated plants showed lower $\mathrm{H}_{2} \mathrm{O}_{2}$ contents than those of the controls (Fig. 2A). Compared with the controls, the increase in $\mathrm{H}_{2} \mathrm{O}_{2}$ content in cut stems treated with AsA

Table 1. Effects of AsA and/or etiolation treatments on morphogenesis and shoot number in tomato plants 9 weeks after cutting stems.

\begin{tabular}{lccc}
\hline \hline \multirow{2}{*}{ Treatment } & \multicolumn{2}{c}{ Morphogenesis (\%) } & \multirow{2}{*}{ Shoot number } \\
\cline { 2 - 3 } & Callus & Adventitious buds & \\
\hline Control & 83 & 17 & $0.1 \mathrm{c}^{\mathrm{z}}$ \\
AsA & 100 & 83 & $13.1 \mathrm{~b}$ \\
Etiolation & 100 & 100 & $33.3 \mathrm{a}$ \\
AsA + Etiolation & 100 & 100 & $33.4 \mathrm{a}$ \\
\hline
\end{tabular}

${ }^{z}$ Different letters indicate significant difference $(P<0.05$; Tukey's multiple range test, $\mathrm{n}=12$ ). was lower at $1 \mathrm{WAC}$, approximately the same at $3 \mathrm{WAC}$, and lower at 4 WAC. In etiolated plants, $\mathrm{H}_{2} \mathrm{O}_{2}$ content transiently increased at $1 \mathrm{WAC}$, and then decreased from 2 WAC and remained at significantly lower levels thereafter. In all tomato plants, the MDA contents in the cut ends of tomato stems increased after cutting (Fig. 2B). However, the MDA contents were significantly lower in those plants that received AsA or etiolation treatments than in the controls from 2 WAC onwards.

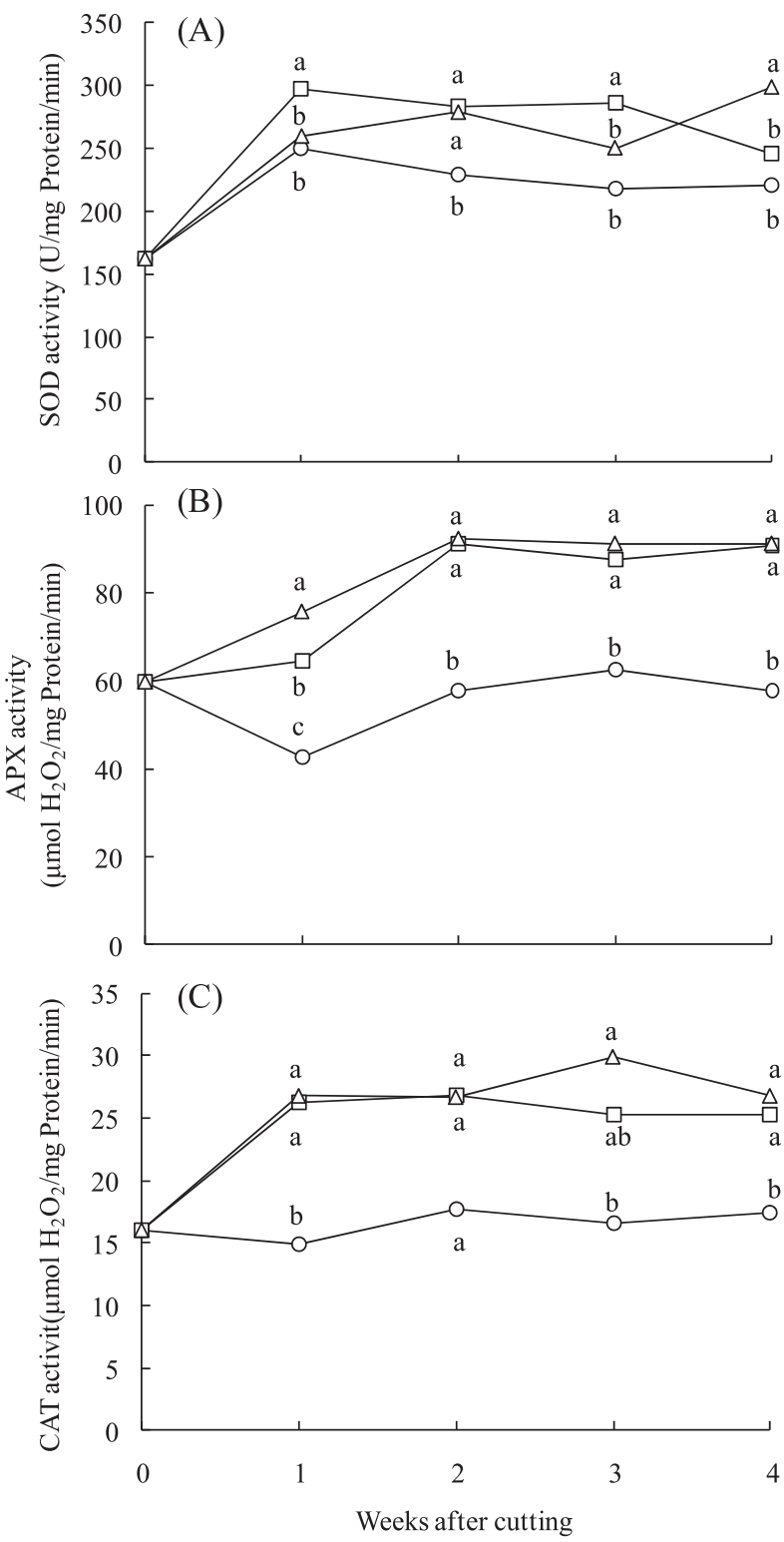

Fig. 1. Effects of ascorbic acid (AsA) or etiolation treatments to cut tomato stems on activities of superoxide dismutase (SOD), ascorbate peroxidase (APX) and catalase (CAT). A, B, and C indicate SOD, APX, and CAT, respectively. $(\bigcirc)$ Control stem; $(\square)$ AsA-treated stem; $(\triangle)$ Etiolated stem. Different letters indicate significant difference $(P<0.05$; Tukey's multiple range test, $\mathrm{n}=3$ ). 

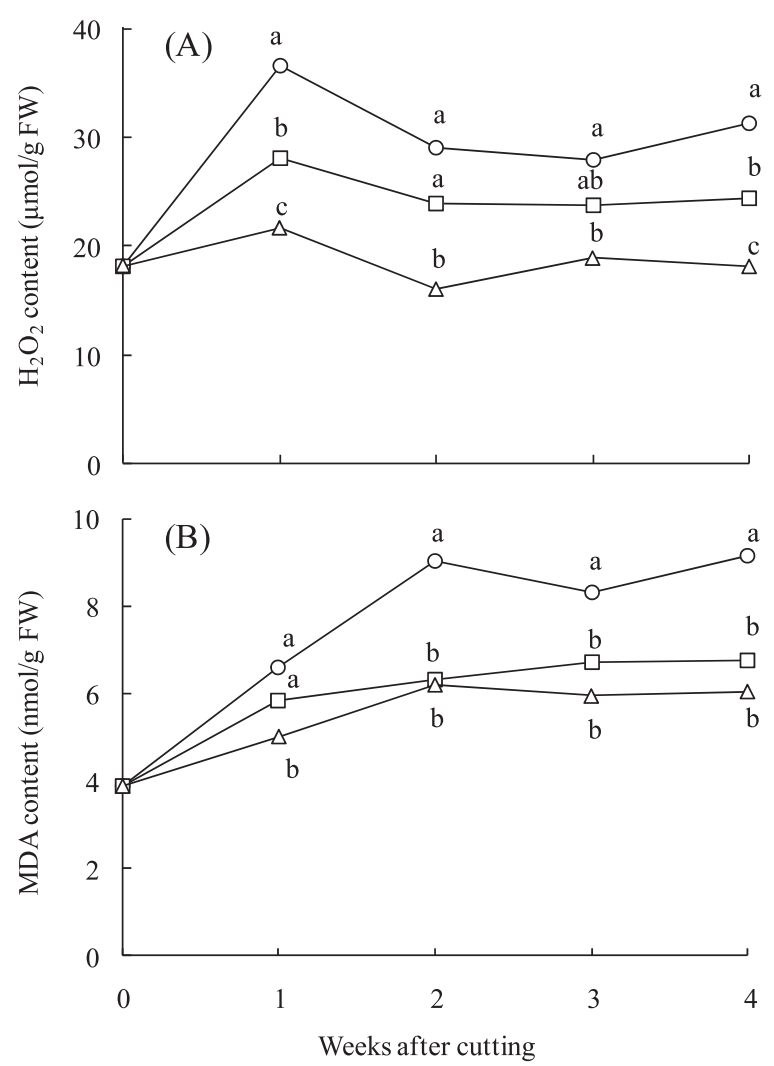

Fig. 2. Effects of ascorbic acid (AsA) or etiolation treatments to cut tomato stems on hydrogen peroxide $\left(\mathrm{H}_{2} \mathrm{O}_{2}\right)$ and malondialdehyde (MDA) contents. $\mathrm{A}$ and $\mathrm{B}$ indicate $\mathrm{H}_{2} \mathrm{O}_{2}$ and MDA, respectively. $(\bigcirc)$ Control stem; $(\square)$ AsA-treated stem; $(\triangle)$ Etiolated stem. Different letters indicate significant difference $(P<0.05$; Tukey's multiple range test, $\mathrm{n}=3)$.

\section{Phenylpropanoid metabolism affected by AsA or etiolation (Exp. 3)}

The activity of PAL, the first enzyme in the phenylpropanoid metabolism, was lower in the cut ends of tomato plants that received AsA or etiolation treatments than in those of the controls (Fig. 3A). PAL activity remained at low levels in the cut ends of tomato plants that received AsA or etiolation treatments. In control plants, PAL activity transiently increased at 1 WAC and then decreased thereafter.

Activities of PPO and POX in the cut ends of tomato stems did not differ among treatments (Fig. 3B, C). In all treatments, the activity of PPO in the cut ends of tomato stems increased at $1 \mathrm{WAC}$ and decreased at 2 WAC, and the activity of POX increased at 1 WAC.

Phenolic content was lower in stems of AsA- or etiolation-treated plants than that in stems of control plants (Fig. 4). After cutting of the stem, the phenol content remained at a low level in stems of plants that received AsA or etiolation treatments, whereas it increased at $1 \mathrm{WAC}$ and then decreased thereafter in stems of control plants.

\section{Discussion}

The promotive effects of AsA or etiolation treatments
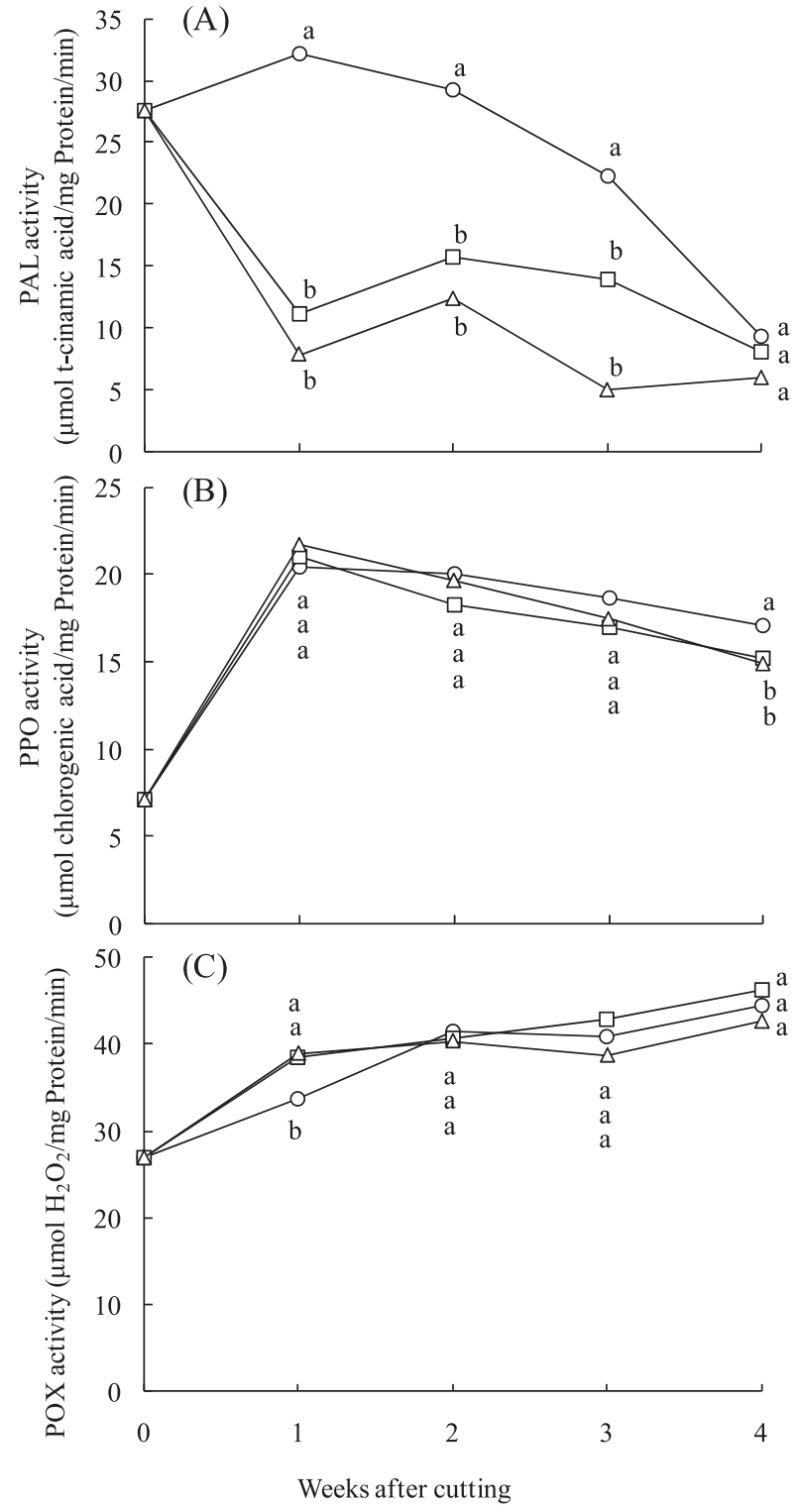

Fig. 3. Effects of ascorbic acid (AsA) or etiolation treatments to cut tomato stems on activities of phenylalanine ammonia-lyase (PAL), polyphenol oxidase (PPO), and peroxidase (POX). A, B, and $\mathrm{C}$ indicate PAL, PPO, and POX, respectively. $(\bigcirc)$ Control stem; ( $\square$ ) AsA-treated stem; $\triangle(\triangle)$ Etiolated stem. Different letters indicate significant difference $(P<0.05$; Tukey's multiple range test, $\mathrm{n}=3$ ).

on shoot regeneration from the cut ends of tomato stems were formed to be similar to those reported previously (Johkan et al., 2008a, b, c, 2009). Application of AsA to plants immediately after stem cutting is a very different treatment from etiolation of the cut stem, but each of these treatments promoted shoot regeneration; therefore, we expected that there might be a synergetic effect of AsA and etiolation. However, our results showed that simultaneous application of both treatments had no synergetic effect, and the shoot regeneration rates were similar between the individual etiolation and simultaneous AsA and etiolation treatment. This result suggests that the promotive effects of AsA or etiolation treatments 


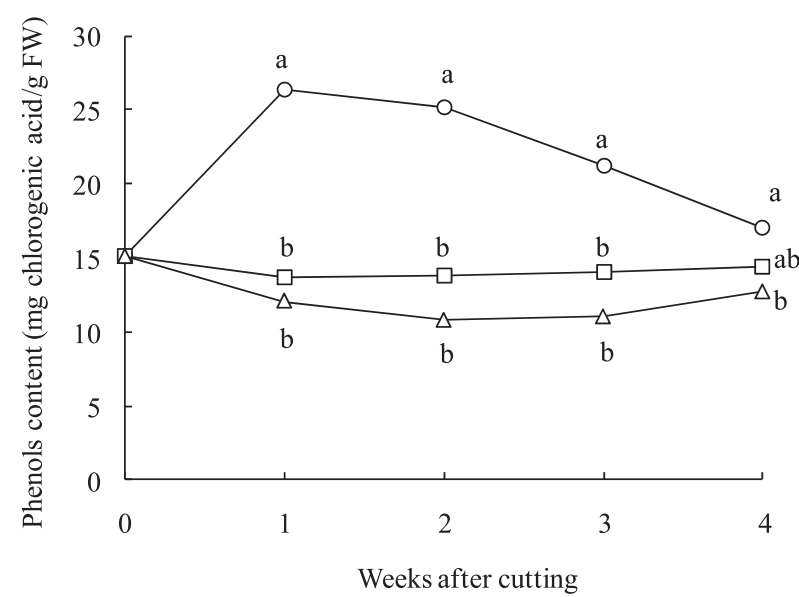

Fig. 4. Effects of ascorbic acid (AsA) or etiolation treatments to cut tomato stems on phenolic contents. $(\bigcirc)$ Control stem; $(\square)$ AsAtreated stem; $(\triangle)$ Etiolated stem. Different letters indicate significant difference $(P<0.05$; Tukey's multiple range test, $\mathrm{n}=3)$.

on shoot regeneration from cut tomato stems might be attributable to the same mechanism.

During the regeneration of shoots from the cut ends of tomato stems, we observed higher activities of antioxidant enzymes such as SOD, APX, and CAT in AsA- or etiolation-treated plants, compared with those in the controls. SOD is a major scavenging enzyme that transforms $\mathrm{O}_{2}{ }^{-}$into $\mathrm{H}_{2} \mathrm{O}_{2}$. The $\mathrm{H}_{2} \mathrm{O}_{2}$ is subsequently transformed into $\mathrm{H}_{2} \mathrm{O}$ via the activities of APX and CAT. Higher activities of antioxidant enzymes were associated with higher rates of cell differentiation and organogenesis in vitro (Qin et al., 2005; Tian et al., 2003). In strawberry tissue culture, the activity of SOD in regenerated calluse increased to 200 times and that of CAT increased to 1.8 times their respective activities in non-regenerated callus (Tian et al., 2003). In control stems, SOD activity increased after cutting, but the activities of APX and CAT were unaffected by cutting. As a result of these lower activities of APX and CAT in the cut ends of control stems, the contents of $\mathrm{H}_{2} \mathrm{O}_{2}$ and MDA were higher than those in stems of plants that received AsA or etiolation treatments. Callus formation had occurred on control stems by 5 WAC, whereas callus formed on those that received AsA or etiolation treatments at 2 or 1 WAC, respectively. These results indicate that higher activities of antioxidant enzymes in the cut ends of tomato stems treated with AsA or etiolation reduced the level of oxidative stress. Moreover, higher activities of antioxidant enzymes, especially APX and CAT, might promote cell differentiation in the cut ends of stems.

In tissue culture, regenerating callus had higher $\mathrm{H}_{2} \mathrm{O}_{2}$ content than non-regenerating callus (Libik et al., 2005; Tian et al., 2003). Libik et al. (2005) suggested that disruption of the oxidative balance could result in excess production of $\mathrm{H}_{2} \mathrm{O}_{2}$, which, in turn, may promote expression of genes responsible for the initiation of morphogenesis. In this study, the contents of $\mathrm{H}_{2} \mathrm{O}_{2}$ and MDA were higher in control stems than in those that received AsA or etiolation treatments. Oxidative stress is imposed by ROS, which are produced in response to cutting of the tomato stems, and lipid peroxidation caused by ROS produces MDA (Qin et al., 2005). Morphogenesis in the cut ends of tomato stems requires oxidative stress to initiate differentiation; however, if the oxidative stress exceeds the antioxidant capacity of the tissues, then shoot regeneration could be suppressed as a result of damage (e.g., lipid peroxidation) to the plant tissues. Therefore, the ability of the AsA or etiolation treatments to increase the activities of antioxidant enzymes (APX, CAT) and thereby decrease oxidative stress may be the reason why these treatments promote early differentiation in tomato stems.

The activities of phenylpropanoid enzymes in the cut ends of tomato stems showed different responses during shoot regeneration from the tomato stem. Patterns of PAL activity and phenol accumulation were similar in the cut ends of tomato plants that received AsA or etiolation treatments. The activity of PAL is affected by oxidative stress, which occurs during physical injury and environmental stress (Dixon and Paiva, 1995), and increased phenol content was correlated with increased PAL activity (Sarma and Sharma, 1999). In the present study, we found that the activities of PPO and POX were similar with all treatments, regardless of the number of shoots that regenerated from the cut ends of tomato stems. PPO, which catalyzes oxidation of mono-phenols and $o$-diphenols (Gil et al., 1998), is related to enzymatic browning (Öztürk and Demir, 2002), and POX is related to lignification of plant tissues (Almagro et al., 2009; Boudet, 2000). Enzymatic browning and lignification restrict regeneration from plant tissues (Kaparakis and Alderson, 2002), but phenols, which are the substrates for enzymatic browning and lignification, were lower in AsA- or etiolation-treated plants than in the control. Therefore, the effects of AsA or etiolation treatments on promoting shoot regeneration from the cut ends of tomato stems might be due to their effects on limiting the accumulation of substrates for enzymatic browning and lignification.

In this study, our results revealed that application of AsA or etiolation treatments to the cut ends of tomato stems increased the shoot number per plant, but there was no synergetic effect when AsA and etiolation treatments were applied simultaneously. In addition, compared with control plants, the tomato plants that received AsA or etiolation treatments showed higher activities of antioxidant enzymes, and lower oxidative stress, PAL activity, and phenolic contents in their cut ends. In tissue culture, oxidative stress occurs in response to injury or environmental stresses imposed by culture conditions, and subsequently results in accumulation of phenols, which are substrates for enzymatic browning and lignification. These responses in the cut ends of 
tomato stems suggested that browning and lignification during the differentiation from callus into other tissues or organs might be unable to respond to high levels of oxidative stress. Fukuoka and Enomoto (2007) reported that lower regeneration in browning Taphanaus roots was associated with increased activities of PAL and PPO, and with impaired activity of the ascorbate-glutathione cycle. In our previous reports, etiolation-treatment prevented the accumulation of phenols (Johkan et al., 2008a), and AsA-treatment scavenged ROS in the cut ends of tomato stems (Johkan et al., 2008b) when shoot regeneration from the cut ends of tomato stems increased. Etiolation treatment suppressed the accumulation of phenolic compounds, which have antioxidant properties, so the increases in activities of antioxidant enzymes in the cut ends of etiolated tomato stems might compensate for the decrease in phenolic compounds. Moreover, AsAtreatment of tomato plants decreased the content of phenols, so the main players in antioxidant capacity may be antioxidant enzymes, rather than phenolic compounds. Unlike in tissue culture, regeneration of shoots from cut stems of tomato plants does not require exogenous hormones. Our results suggest that suppression of oxidative stress by increased activities of antioxidant enzymes, which occurred in cut ends of AsAor etiolation-treated tomato plants, might be an important factor in the regeneration of shoots from the cut ends of tomato stems.

In conclusion, etiolation treatments increased the number of shoots regenerated from the cut ends of tomato stems, irrespective of AsA treatment. The promotive effects of individual AsA- or etiolation treatments on shoot regeneration from the cut ends of tomato stems were attributed to the same mechanism. This mechanism involved high activities of antioxidant enzymes, low oxidative stress, and low phenolic contents in the cut ends of tomato stems.

\section{Literature Cited}

Almagro, L., L. V. Gomez, S. Belchi-Navarro, R. Bru, A. Ros Barcelo and M. A. Pedreno. 2009. Class III peroxidases in plant defence reactions. J. Exp. Bot. 60: 377-390.

Boudet, A. M. 2000. Lignins and lignification: Selected issues. Plant Physiol. Biochem. 38: 81-96.

Bradford, M. M. 1976. A rapid and sensitive method for the quantitation of microgram quantities of protein utilising the principle of protein-dye binding. Anal. Biochem. 72: 248254.

Dipierro, S. and S. D. Leonardis. 1997. The ascorbate system and lipid peroxidation in stored potato (Solanum tuberosum L.) tubers. J. Exp. Bot. 48: 779-783.

Dixon, R. A. and N. L. Paiva. 1995. Stress-induced phenylpropanoid metabolism. Plant Cell 7: 1085-1097.

Dogan, S., P. Turan, M. Dogan, O. Arslan and M. Alkan. 2007. Variations of peroxidase activity among Salvia species. J. Food Engin. 79: 375-382.

Feng, B., Y. Chen, C. Zhao, X. Zaho, X. Bai and Y. Du. 2006. Isolation of a novel Ser/Thr protein kinase gene from oligochitosan-induced tobacco and its role resistance against tobacco mosaic virus. Plant Physiol. Biochem. 44: 596-603.

Fukuoka, N. and T. Enomoto. 2007. Effects of sulfur application on enzyme activities in relation to the ascorbate-glutathione cycle and the occurrence of internal browning. J. Japan. Soc. Hort. Sci. 76: 305-309.

Gil, M. I., J. R. Gorny and A. A. Kader. 1998. Responses of 'Fuji' apple slices to ascorbic acid treatments and low-oxygen atmospheres. HortScience 33: 305-309.

Gupta, S. D. and S. Datta. 2003. Antioxidant enzyme activities during in vitro morphogenesis of gladiolus and the effect of application of antioxidants on plant regeneration. Biol. Plant 47: 179-183.

Harada, M., M. Oda, G. Mori and H. Ikeda. 2005. Mass regeneration of shoots from cut surface of stems in tomato stock plants. J. Japan. Soc. Hort. Sci. 74: 479-481.

Johkan, M., G. Mori, K. Mitsukuri, K. Mishiba, T. Morikawa and M. Oda. 2008a. In vivo shoot regeneration promoted by shading the cut surfaces of the stem in tomato plants. HortScience. 43: 1-4.

Johkan, M., G. Mori, K. Mitsukuri, K. Mishiba, Y. Imahori, T. Morikawa and M. Oda. 2008b. Effect of ascorbic acid on in vivo organogenesis in tomato plants. J. Hort. Sci. Biotech. 83: 624-628.

Johkan, M., G. Mori, Y. Imahori, K. Mitsukuri, S. Yamasaki, K. Mishiba, T. Morikawa and M. Oda. 2008c. Shading the cut stems of tomato plants promotes in vivo shoot regeneration via control of the phenolic metabolism. Environ. Cont. Biol. 46: 203-209.

Johkan, M., M. Oda, K. Mitsukuri and S. Yamasaki. 2009. Effect of etiolation on in vivo shoot regeneration in tomato plants at different cultivars and seasons. Hort. Environ. Biotech. 50: 208-212.

Kaparakis, G. and P. G. Alderson. 2002. Influence of high concentrations of cytokinins on the production of somatic embryos by germinating seeds of tomato, aubergine and pepper. J. Hort. Sci. Biotech. 77: 186-190.

Kawakami, S., M. Mizuno and H. Tsuchida. 2000. Comparison of antioxidant enzyme activities between Solanum tuberosum L. cultivar danshaku and kitaakari during low-temperature storage. J. Agric. Food Chem. 48: 2117-2121.

Libik, M., R. Konieczny, B. Pater, I. Ślesak and Z. Miszalski. 2005. Differences in the activities of some antioxidant enzymes and in $\mathrm{H}_{2} \mathrm{O}_{2}$ content during rhizogenesis and somatic embryogenesis in callus cultures of the ice plant. Plant Cell Rep. 23: 834-841.

Mitsukuri, K., G. Mori, M. Johkan, K. Mishiba, T. Morikawa and M. Oda. 2009. Effects of explant source and darkpreconditioning on adventitious bud formation in Neofinetia falcate H. H. Hu in vitro. J. Japan. Soc. Hort. Sci. 78: 252-256.

Murata, M., M. Tsurutani, M. Tomita, S. Homma and K. Kaneko. 1995. Relationship between apple ripening and browning: changes in polyphenol content and polyphenol oxidase. J. Agric. Food Chem. 43: 1115-1121.

Nakano, Y. and K. Asada. 1981. Hydrogen peroxide is scavenged by ascorbate-specific peroxide in spinach chloroplasts. Plant Cell Physiol. 22: 867-880.

Oda, M., M. Y. Huang, H. Ikeda and H. Furukawa. 2008. Effects of cultivar, decapitation and training direction on the uniformity and number of lateral shoots in the vegetative propagation of tomato plants. J. Sci. High-Tech. Agr. 20: 152-157 (In Japanese with English abstract).

Okuda, T., Y. Masuda, A. Yamanaka and S. Sagisaka. 1991. Abrupt increase in the level of hydrogen peroxide in leaves of winter wheat is caused by cold treatment. Plant Physiol. 97: 12651267. 
Öztürk, L. and Y. Demir. 2002. In vivo and in vitro protective role of proline. Plant Growth Regul. 38: 259-264.

Padmanabhan, V., E. P. Paddock and W. R. Sharp. 1974. Plant formation from Lycopersicon esculentum leaf callus. Can. J. Bot. 52: 1429-1432.

Qin, Y., S. Zhang, A. Syed, L. Zhang, Q. Qin, K. Chen and C. $\mathrm{Xu}$. 2005. Regeneration mechanism of Toyonoka strawberry under different color plastic films. Plant Sci. 168: 1425-1431.

Sarma, A. D. and R. Sharma. 1999. Purification and characterization of UV-B induced phenylalanine ammonialyase from rice seedlings. Phytochemistry 50: 729-737.

Sharma, R. R. and S. K. Singh. 2002. Etiolation reduces phenolic content and polyphenol oxidase activity at the pre-culture stage and in-vitro exudation of phenols from mango explants. Trop. Agric. 79: 94-99.

Shirai, T. and M. Hagimori. 2004a. A multiplication method of sweet pepper (Capsicum annuum L.) by vegetative propagation. J. Japan. Soc. Hort. Sci. 73: 259-265 (In
Japanese with English abstract).

Shirai, T. and M. Hagimori. 2004b. Multiplication method of Capsicum annuum L. by soilless culture: Effects of planting density of mother plants and the substrates on yield of shoots. J. Japan. Soc. Hort. Sci. 73: 374-379 (In Japanese with English abstract).

Shirai, T. and M. Hagimori. 2004c. Studies in establishment of transplant production method of sweet pepper (Capsicum annuum L.) by grafting shoots harvested from mother plants: Effect of healing condition of grafts on the rate and quality of successful union. J. Japan. Soc. Hort. Sci. 73: 380-385 (In Japanese with English abstract).

Singleton, V. L. and J. A. Rossi. 1965. Colorimetry of total phenolics with phosphomolybedic-phosphototungstic acid reagents. Amer. J. Enol. Viticult. 16: 144-158.

Tian, M., Q. Gu and M. Y. Zhu. 2003. The involvement of hydrogen peroxide and antioxidant enzymes in the process of shoot organogenesis of strawberry callus. Plant Sci. 165: 701-707. 